\title{
Un ECG typique de tako-tsubo
}

\section{Typical ECG of tako-tsubo}

\section{P. Taboulet}

Reçu le 1 mai 2013 ; accepté le 5 juin 2013

(C) SFMU et Springer-Verlag France 2013

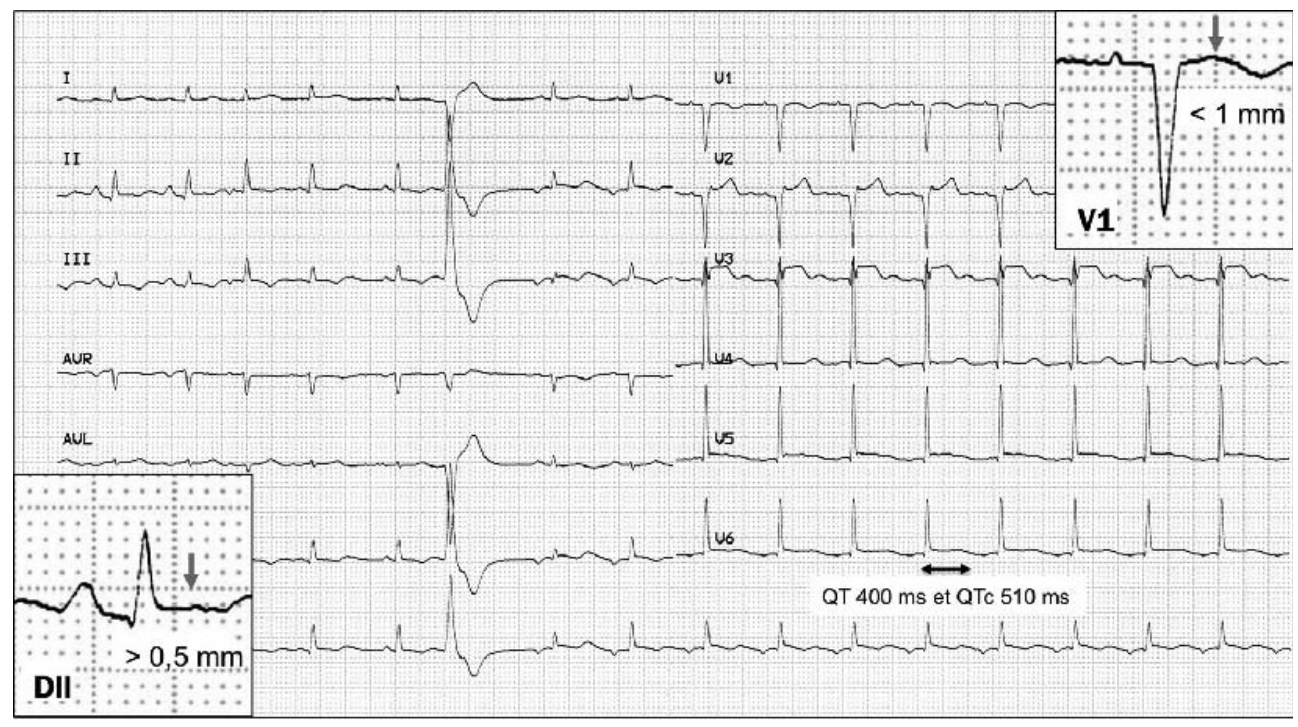

Fig. 1 Tako-tsubo. Notez le sus-décalage de ST en DII et l'absence de sus-décalage de ST en V1 (mesures à 80 ms du point J). Les troubles de la repolarisation sont plutôt diffus et peu amples avec prolongation de l'intervalle QTc

Une femme de 59 ans consulte aux urgences pour douleur abdominale, fluctuante, non spécifique depuis environ huit heures. L'examen clinique est non spécifique, les paramètres vitaux normaux, mais la découverte d'une hyperglycémie à $15,8 \mathrm{mmol} / \mathrm{l}$ conduit à réaliser un ECG (Fig. 1). Celui-ci inscrit un rythme d'abord sinusal, puis un rythme atrial ectopique. Les ondes Q septales et les troubles de repolarisation font évoquer un infarctus antérieur non datable ou un takotsubo (cardiomyopathie aiguë catécholergique ou « infarctus de stress à coronaires saines »). En faveur du tako-tsubo, on observe en dérivation DII, en rythme sinusal, un sus-décalage du segment ST $>0,5 \mathrm{~mm}$ et en dérivation $\mathrm{V} 1$ une absence de sus-décalage de ST (inhabituels pour un infarctus ST + anté-

P. Taboulet $(\square)$

Hôpital Saint-Louis,

Assistance publique-hôpitaux de Paris,

service des urgences, 1, avenue Claude Vellefaux,

F-75010, Paris, France

e-mail : pierre.taboulet@sls.aphp.fr rieur). Dans la série de Kosuge et al. [1], cette association avait une valeur prédictive positive $>90 \%$ en faveur d'un tako-tsubo. En effet, V1 explore davantage la base du ventricule gauche que DII (et -VR) qui explore(nt) davantage l'apex, plus affecté par le tako-tsubo que la base. D'autres signes sont en faveur du tako-tsubo : les ondes $\mathrm{Q}$ sont profondes dès l'ECG initial, le nombre de dérivations avec susdécalage de ST est élevé, la déviation du segment ST est modérée et plutôt concave, le miroir en territoire inférieur est absent, les ondes T sont peu amples ou inversées et l'intervalle QTc est plus long qu'au cours d'un infarctus [1]. Chacun de ses signes peut se voir au cours d'un syndrome coronaire aigu, mais tous réunis, ils évoquent davantage un tako-tsubo.

\section{Référence}

1. Kosuge M, Ebina T, Hibi K, et al (2010) Simple and accurate electrocardiographic criteria to differentiate takotsubo cardiomyopathy from anterior acute myocardial infarction. J Am Coll Cardiol $55: 2514-6$ 